\title{
A Randomized Blinded Trial of Nucleus Accumbens Ablation to Treat Opiate Dependence in Humans: Location Correlates with Outcome
}

Xuelian Wang ${ }^{1}$, Chongwang Chang ${ }^{1}$, John R. Adler ${ }^{2}$, Nan Li $^{1}$, Shunnan Ge ${ }^{1}$, Ning Geng ${ }^{1}$, Jing Wang ${ }^{1}$, Xin Wang ${ }^{1}$, Guodong Gao ${ }^{3}$

1. Department of Neurosurgery, Tangdu Hospital, Xi'an, China 2. Department of Neurosurgery, Stanford University School of Medicine 3. Department of Neurosurgery, Tangdu Hospital, Xi'an, China, Department of Neurosurgery, Tangdu Hospital, Xi'an, China

$\square$ Corresponding author: Xuelian Wang, tdwxlian@126.com

Disclosures can be found in Additional Information at the end of the article

\section{Abstract}

Background: Opiate addiction remains intractable in a large percentage of patients. Multiple studies identify a central role of the nucleus accumbens (NAc) in addiction; several studies note decreased addictive behavior after interventions in this area. In China, ablation of the NAc was common before November 2004, though it was poorly regulated. This study, a prospective randomized double-blinded trial carried out from January 2004 to November 2004, investigates the effect of different stereotactic lesions within NAc on opiate addiction to maximize benefit and minimize risk.

Methods: Seventy-eight opiate-dependent patients were treated at the Tangdu Hospital Fourth Military Medical University during Jan. 2004 to Nov. 2004 with bilateral stereotactic radiofrequency lesioning of the posteromedial one-third of NAc. Patients were randomly divided into four groups (A, B,C, and D). Relative to the initial target, the lesion in Group A was enlarged laterally and superiorly, in Group B anteriorly and superiorly, and in Group C laterally and anteriorly. Among these three groups, the lesion volume was identical. In Group D, the lesion extended laterally and anteriorly and superiorly. Using opiate abstinence as the primary endpoint, four years of follow-up data for all patients regarding relapse and complications were collected. This trial is supplementarily registered with Current Controlled Trials, number ISRCTN33954423.

Results: Four patients were withdrawn at the fourth year post-operation. For convenience of interpretation, this article appointed these patients as relapses. The overall abstinence rate was $53.8 \%$ (42/78). The abstinence rates of Group C (75.0\%) and Group D (76.2\%) were higher than Groups A (31.3\%) and B (28.6\%) ( $\mathrm{p} \leqslant 0.005)$. The neuropsychological adverse event rate was $24.3 \%(18 / 74)$ and did not differ statistically between the four groups.

Published 06/07/2012

\section{(๑) Copyright 2012}

Wang et al. This is an open access article distributed under the terms of the Creative Commons Attribution License CC-BY 3.0., which permits unrestricted use, distribution, and reproduction in any medium, provided the original author and source are credited.
Conclusions: Although sometimes accompanied by neuropsychological adverse events, stereotactic ablation of NAc may effectively treat opiate addiction. Lesion location significantly impacts treatment efficacy.

Categories: Neurology, Psychology, Neurosurgery

Keywords: surgical procedures, nucleus accumbens, stereotactic neurosurgery, opiate addiction, 


\section{Introduction}

There exists a compelling need for effective treatments for severe neuropsychiatric diseases, particularly refractory heroin addiction, where effective options are limited. Many interventions are aimed at heroin use reduction, rather than cessation. Among the most successful is the use of methadone for detoxification or maintenance therapy; even within these programs, greater than $50 \%$ continue to use heroin [1-3]. Chinese centers employing compulsory detoxification, mandatory labor, education, skills training, and lasting for as long as three years, report a one year abstinence rate of $44 \%$, and a three year abstinence rate of only $15 \%$ [4]. However, the addition of pharmacologic interventions may significantly improve these rates. Nevertheless, a randomized control trial of Malaysian patients taking naltrexone or buprenorphine demonstrated average time to relapse of 61 and 79 days, respectively [5]. Meanwhile, one Italian longitudinal study of over 5,000 patients demonstrated at six months only a 50\% treatment retention rate [6]. Despite multiple treatment modalities, the majority of heroin users continue to use, which not only harms the patient but also presents risks to public health, with increased spread of blood-borne pathogens, such as hepatitis C and HIV.

The inability to provide long-term relief for many patients suffering from severe neuropsychiatric disease, combined with an increasing knowledge of neural circuitry and a modular understanding of the human brain, has renewed interest in surgical interventions for these diseases. The mesolimbic dopamine system plays a central role in brain reward mechanisms with the nucleus accumbens (NAc) serving as a key structure in mediating these effects [7-10]. Based on this understanding, some functional neurosurgeons have used ablation or stimulation of the NAc to effectively treat severe refractory cases of obsessive-compulsive disorders and depression [11-21]. These patients incidentally noted decreases in chemical dependence [15-16]. In parallel, a series of Chinese animal studies suggested the utility of NAc lesioning in treating drug dependence [22], which led to initial trials in humans in China with reasonable efficacy [11].

Given the important role this and surrounding structures play in defining personality and human motivation, serious attention must be paid to neuropsychiatric complications from these interventions. Prior data on nucleus accumbens ablation suggests no change in intelligence postoperatively, though psychological testing indicates deficiencies in short-term memory, attention, and possibly some personality characteristics, though long-term follow-up was not presented [23].

The NAc is a critical part of the mesocorticolimbic dopaminergic system, receiving input from ventral tegmental area and directing outputs to the prefrontal cortex. It has long been implicated in substance abuse and addiction, including ethanol, cocaine, and heroin [16-17, 2533].

Opioid receptors in the NAc likely play a key role in the pathophysiology of all addiction, especially opiates [34-39]. The shell and core of the NAc may play differing roles; among other differences, they project to different targets. Injection of opioid receptor blockers into the NAc shell in heroin-exposed rats appears to decrease heroin use [40]. The shell demonstrates different expression profiles depending on whether the heroin exposure was self-administered, suggesting a differential role of the NAc shell in psychological addiction [25]. Ablation of the core in morphine-addicted rats impaired both morphine and electroshock place conditioning, while shell lesions were no different than sham lesions [41]. Further research may help identify and shape future targets to effectively abate psychological addiction.

Surgical interventions within the nucleus accumbens are reported to have variable rates of 
efficacy and complications for a range of neuropsychiatric illnesses [11-12, 14, 16, 19, 21, 23]. Some of this variability may stem from differing surgical techniques for ablating nucleus accumbens among different medical centers; even within a given technique, in this case, radiofrequency ablation of the nucleus accumbens, widely varying complications have been reported, resulting in significant controversy [24]. We hypothesize that slight variations in lesion location may have an important influence on clinical outcome. To investigate the optimal lesion site, we established a prospective randomized double-blinded trial to analyze opiate abstinence and complication rate in different radiofrequency ablation lesion locations within the NAc.

\section{Materials And Methods}

\section{Trial design}

This study was examined and approved by the Ethics Committee of Fourth Military Medical University. The trial is a randomized, prospective, double-blinded clinical trial with planned enrollment of 80 patients. Subjects were randomized to four groups and followed at six month intervals for four years. Primary endpoint was abstinence from opiate use. Neuropsychological adverse events were also tracked. This trial had been supplementarily registered with Current Controlled Trials, number ISRCTN33954423.

\section{Participants}

All opiate-dependent patients involved in this study presented to the department from January 2004 to November 2004 for consideration of bilateral nucleus accumbens ablation. Inclusion criteria were: (1) heroin abuse using $0.3-1.0 \mathrm{~g}$ daily for at least three years by intravenous injection with or without concomitant nasal inhalation, (2) failure of multiple other treatment modalities and with five or more relapses; (3) age between 18 and 50; (4) no surgical contraindication; (5) completion of detoxification treatment (the overall detoxification period no less than one year) preoperatively with (a) no somatic symptoms of withdrawal and (b) negative morphine urinalysis and naloxone tests. The exclusion criteria included: (1) inability to give informed consent; (2) HIV, HBV or HCV virus carrier; (3) developmental delay, cognitive impairment, personality disorders, and neuropsychiatric diseases, other than addiction. In addition, patients had to agree to undergo no additional treatment for opiate addiction postoperatively, including counseling. Every patient and, where appropriate, guardian, was thoroughly informed of the risks and benefits of the proposed operation and gave written informed consent to participate in the study. Subjects were randomized into four groups as described below. Final follow-up was conducted at the university. For patients unable to travel to the university, a team of researchers were sent to the patient's home.

\section{Surgical procedures}

After establishing the patient to be free of opiate use and physiologic dependence, as confirmed by a negative urinalysis and naloxone testing, patients underwent radiofrequency ablation of the NAc. The surgical procedure began by affixing the Cosman-Robert-Wells (CRW) stereotactic ring (Radionics Inc., Burlington, Mass, USA) to the patient's head under local anesthesia, making the base parallel to the projected line of the anterior and posterior commissure. Patients then underwent MRI imaging using an axial T-2 weighted sequence with a $3 \mathrm{~mm}$ slice thickness in a GE 1.0T magnet. Imaging data was reconstructed in three-dimensions using Easyvision software (Philips Medical Systems). To improve localization of the nucleus accumbens in standard MRI images, segmentation methods devised by The Center for Morphometric Analysis at Harvard Medical School (http://www.cma.mgh.harvard.edu/) were utilized. The borders of the NAc were determined as follows: 1) the most anterior or rostral margin of the NAc was defined as the point where the caudate and putamen are first separated by the internal capsule, 2) the posterior or caudal margin was defined as the anterior border of 
anterior commissure and gyrus subcallosus, 3) the superior or dorsal margin was defined by the inferior portions of the internal capsule, caudate and putamen, 4) the inferior or ventral border was defined by Broca's diagonal band and the white matter fiber of the external capsule, 5) the medial border was defined by the septal nuclei, and finally, 6) the lateral border was defined by the lateral ventricle and the medial border of putamen. Using the above segmentation method, the location of the initial lesion loci were standardized according to the Schaltenbrand-Bailey standard human brain atlas. The initial lesion was made at a point 6.0-8.0 mm inferior and 17$22 \mathrm{~mm}$ anterior and 5.0-6.5 mm lateral to the midpoint of a line drawn between the anterior commissure and posterior commissure at midline. This lesion location corresponded to the posteromedial one-third portion of the nucleus accumbens.

Bilateral frontal incisions, located 2.0-2.5 cm lateral to the midline, were made under local anesthesia. The ablation was performed by the RFG-3CF Radiofrequency Lesion Generator (Radionics, Burlington, MA, USA) with an electrode (1.6 mm in diameter, $4 \mathrm{~mm}$ exposed electrode). After stereotactically placing the electrode tip at the target, a low frequency (2 Hz) followed by a high-frequency $(100 \mathrm{~Hz})$ stimulus was applied using an amplitude of 6-8 V. Patients underwent hemodynamic and neurologic monitoring, including observation by the surgeon and a nurse specialist. In addition to standard monitoring, patients were specifically observed for fever, diaphoresis, palpitations, headache, dyspnea, dysphasia, paresis, seizure, paresthesia, dysphoria, and akisthesia. A preliminary lesion $\left(45^{\circ} \mathrm{C}, 60\right.$ seconds) was then made. If no abnormal reaction was observed, a definitive radiofrequency lesion $\left(80^{\circ} \mathrm{C}, 60\right.$ seconds) was carried out.

As described above, patients were randomized into one of four different groups (Group A, B, C, and D). The lesioning procedures were as follows: Group A: four foci were lesioned, the first at the initial point, the second four mm superior, the third focus three mm lateral to the first one, and the fourth four mm superior to the third (Figure 1A). Group B (Figure 1B): four foci were lesioned, the first at the initial point, the second four mm superior, the third focus three $\mathrm{mm}$ anterior to the first one, and the fourth four mm superior to the third. Group C (Figure 1C): four foci were lesioned, the first at the initial point, the second four mm superior, the third focus three mm lateral to the first one, and the fourth three mm anterior to the first. Group D (Figure 1D): six foci were lesioned, the first at the initial point, the second four mm superior, the third focus three mm lateral to the first one, the fourth focus four mm superior to the third, the fifth three mm anterior to the first, and the sixth four mm superior to the fifth. The accuracy of targeting was radiographically confirmed in all cases with postoperative MRI. 


\section{Cureus}
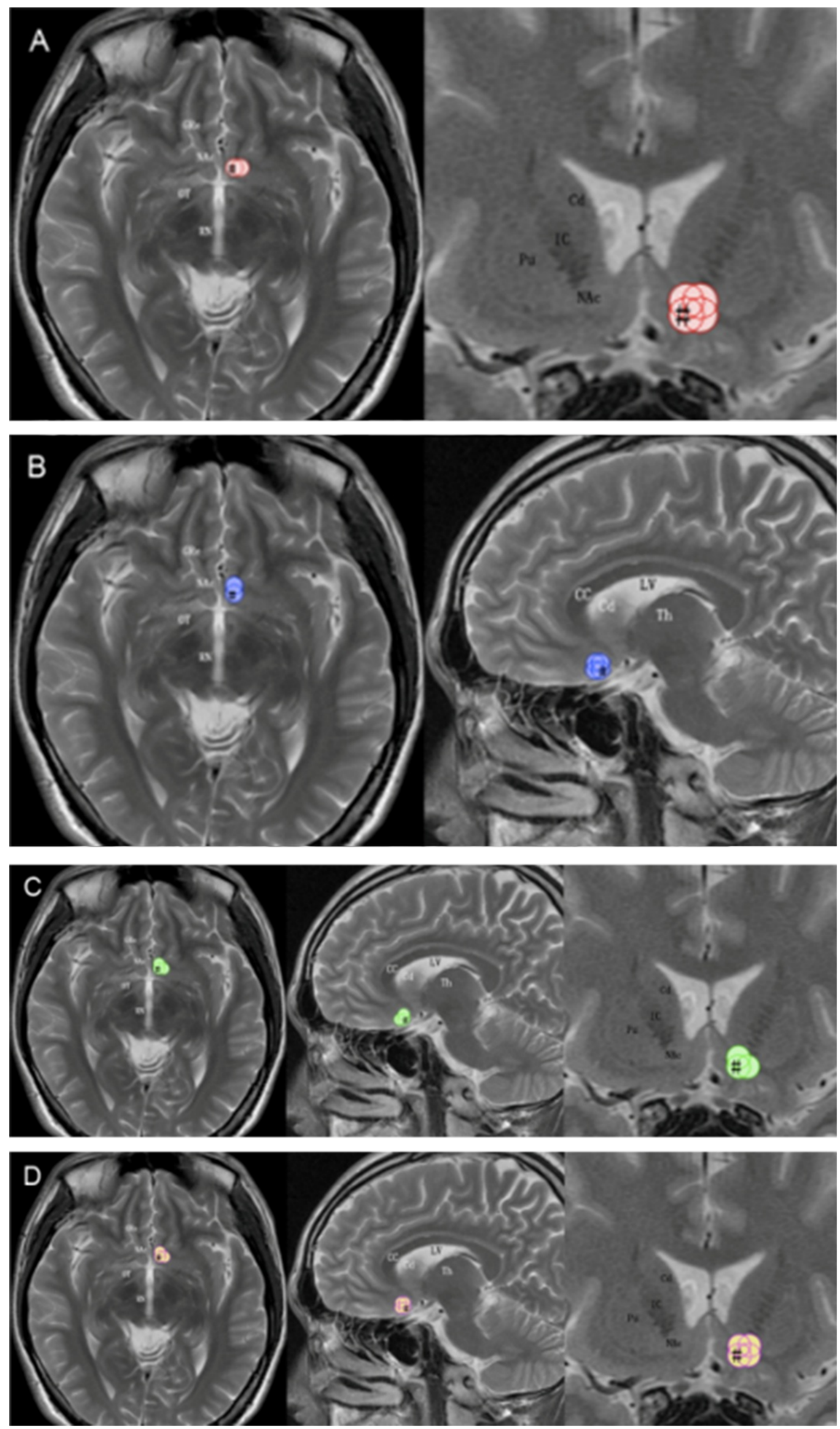

FIGURE 1: The schematic diagram of lesion loci. 
A. Group A (axial and coronal views). CC: corpus callosum; Cd: caudate nucleus; GRe: gyrus rectus; Ic: internal capsule; LV: lateral ventricle; NAc: Nucleus accumbens; OT: optical tract; Pu: putamen; RN: red nucleus; Th: thalamus. \#indicates initial lesion. B. Group B (axial and sagittal views). CC: corpus callosum; Cd: caudate nucleus; GRe: gyrus rectus; Ic: internal capsule; LV: lateral ventricle; NAc: Nucleus accumbens; OT: optical tract; Pu: putamen; RN: red nucleus; Th: thalamus. \# indicates initial lesion. C. Group C (axial, sagittal and coronal views). CC: corpus callosum; Cd: caudate nucleus; GRe: gyrus rectus; Ic: internal capsule; LV: lateral ventricle; NAc: Nucleus accumbens; OT: optical tract; Pu: putamen; RN: red nucleus; Th: thalamus. \# indicates initial lesion. D. D group (axial, sagittal and coronal views). CC: corpus callosum; Cd: caudate nucleus; GRe: gyrus rectus; Ic: internal capsule; LV: lateral ventricle; NAc: Nucleus accumbens; OT: optical tract; Pu: putamen; RN: red nucleus; Th: thalamus. \# indicates initial lesion.

\section{Outcomes and evaluation}

The primary outcome measure was abstinence from opioid use. Patients, clinical staff, and statistical clerks were blinded to both the research objectives and patients' lesion status. Neither study designers nor surgeons participated in the postoperative follow-up and results analysis. Postoperative follow-up and evaluation were performed by two neuropsychologists (including N.G.) who each had more than two years clinical experience in addiction medicine. Follow-up was conducted at six month intervals and extended for four years for all patients. The follow-up methods during this period included outpatient interviews, telephone inquiry, and letter correspondence. In the fourth postoperative year, every patient returned to our department and extensive data were collected through face-to-face interviews and examination, including morphine urinalysis. Regardless of any contradictory data, positive morphine urinalysis was considered a relapse. If morphine urinalysis was negative, naloxone testing was performed. Positive naloxone testing was also considered a relapse, regardless of other data. Follow-up data included a range of complications, such as anterograde amnesia, motivational dysfunction, affective disorders, and dysophresia. Formal neuropsychiatric instruments were employed: for memory, the Wechsler Memory Scale-Revised for China (WMSRC); for motivation: Academic Achievement Scale (Ac) and the Counselor Personality Scale (Cs); for emotion: the N factor of the Eysenck Personality Questionnaire (EPQ-N), the C factor of the Cartel 16 Personality Factor Test (16PF-C) and ANG scaling of the Minnesota Multiphasic Personality Inventory (MMPI-ANG); for olfactory sensation: the Connecticut Chemosensory Clinic Research Center Test (CCCRC). Adverse events were identified by patient or family complaint, or by clinical impression of the treating physician. Formal neuropsychiatric instruments were used to quantify the magnitude of pre- and post-operative changes over all patients. All patients underwent formal neuropsychiatric testing regardless of subjective impressions.

\section{Statistical analysis}

Statistical analysis of all data was done using SPSS 13.0 software. The $\chi^{2}$-test was applied for difference analysis, setting $\alpha=0.05$ to be fundamental test size. In applying the $\chi^{2}$-test to measured differences between the separate experimental groups, significance was measured at $\alpha=0.0125$. Using correlated samples t-test to analysis the difference of the pre- and postneuropsychiatric changes. The statistical analysis was reviewed and endorsed by statisticians at Stanford University Hospitals and Clinics.

\section{Results}

\section{Demographics}




\section{Cureus}

\begin{tabular}{|c|c|c|c|c|c|c|c|c|}
\hline \multirow{2}{*}{ Group } & \multirow{2}{*}{ Cases } & \multirow{2}{*}{ Male } & \multirow{2}{*}{ Female } & \multirow{2}{*}{$\begin{array}{l}\text { Average } \\
\text { age (years old) }\end{array}$} & \multirow{2}{*}{$\begin{array}{l}\text { Drug use } \\
\text { history (years) }\end{array}$} & \multirow{2}{*}{$\begin{array}{l}\text { Mean daily } \\
\text { dose (g) }\end{array}$} & \multicolumn{2}{|c|}{ Administration method } \\
\hline & & & & & & & miliaverious & Transnasal with intravenous \\
\hline A & 16 & 15 & 1 & $26.3 \pm 4.2$ & $3.8 \pm 1.3$ & $0.5 \pm 0.2$ & 10 & 6 \\
\hline B & 21 & 19 & 2 & $26.1 \pm 3.3$ & $4.5 \pm 1.2$ & $0.5 \pm 0.4$ & 14 & 7 \\
\hline C & 20 & 19 & 1 & $27.2 \pm 3.4$ & $3.9 \pm 1.2$ & $0.6 \pm 0.3$ & 14 & 6 \\
\hline D & 21 & 21 & 0 & $26.8 \pm 3.6$ & $3.7 \pm 1.4$ & $0.5 \pm 0.3$ & 14 & 7 \\
\hline
\end{tabular}

\section{TABLE 1: Clinical data of cases in four groups $(\mathrm{N}=78)$}

Notes: There were no significant differences between patient groups on above variables.

The study ceased when reports of procedural misuse from other centers caused the China's Ministry of Public Health to ban the surgery outright and only 78 patients (Flow Diagram) were enrolled. Two patients were excluded from analysis for seeking further treatment (residentialbased treatment); one patient refused face-to-face interviews; and one patient was lost to follow-up. Therefore, complete data, including complications, were available for 74 patients. Patients who withdrew were considered treatment failures, and thus, the major outcome abstinence includes 78 patients.

The final study group included 74 males and four females whose ages ranged from 21 to 47 years old, with an average age of $26.7 \pm 3.9$ years old. There were 16 patients in Group A, 21 patients in Group B, 20 patients in Group C, and 21 patients in Group D. There were no significant differences among the four groups with respect to sex, age, duration, method of administration, or quantity of drug abuse (P区0.05), as shown in Table 1. 


\section{Cureus}
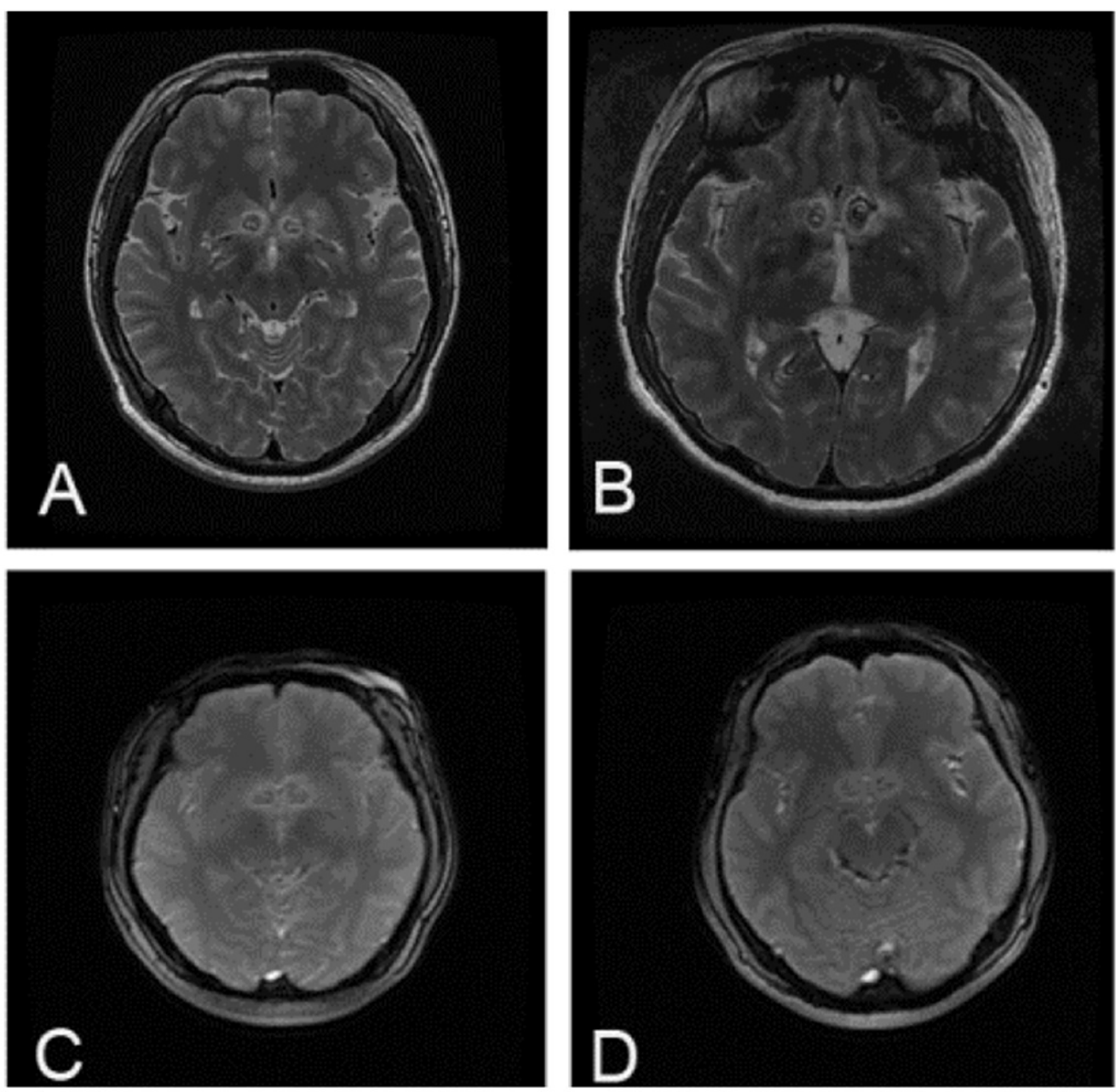

\section{FIGURE 2: The postoperative MRI images of patients.}

The postoperative MRI scanning showed the condition of lesion foci of four patients belonging to four surgical groups. A. MRI of one patient in Group A (axial views, T2-weighted image). B. MRI of one patient in Group B (axial views, T2-weighted image). C. MRI of one patient in Group C (axial views, T1-weighted image). D. MRI of one patient in Group D (axial views, T1-weighted image).

The postoperative MRI scanning showed the condition of lesion foci of four patients belonging to four surgical groups. A: MRI of one patient in Group A (axial views, T2-weighted image). B: MRI of one patient in Group B (axial views, T2-weighted image). C: MRI of one patient in Group C (axial views, T1-weighted image). D: MRI of one patient in Group D (axial views, T1-weighted image) and four females whose ages ranged from 21 to 47 years old, with an average age of $26.7 \pm 3.9$ years old. There were 16 patients in Group A, 21 patients in Group B, 20 patients in Group C, and 21 patients in Group D. There were no significant differences among the four groups with respect to sex, age, duration, method of administration, or quantity of drug abuse (Pख0.05), as shown in Table 1.

\section{Examination of lesion locus}

The position of lesion foci was inspected by postoperative MRI for all patients. All the results revealed that the locus was accurate and was in line with the certain surgical procedures for different surgical groups (Figure 2). Further analysis of this data is ongoing. 


\section{Cureus}

\section{Primary outcome: Abstinence}

The total abstinence rate of all patients at their fourth post-operation year was $53.8 \%(42 / 78)$. At four years, Groups C (75.0\%) and D (76.2\%) had much higher abstinence rates than groups A (31.3\%) or B (28.6\%) ( $\mathrm{p}<0.005)$ (Table 2 and Figure 3). The concordance rate of results from follow-up questionnaires filled out by patients or their guardians was $95.3 \%$.

\section{Items}

Total patients

Abstinent patients and abstinence rate

\begin{tabular}{l|l|l|l|l|}
\hline Group A & Group B & Group C & Group D & P \\
\hline 16 & 21 & 20 & 21 & \\
\hline $5(31.3 \% \square$ & $6(28.6 \% \square$ & $15(75.0 \% \square$ & $16(76.2 \%)$ & $<0.005$ \\
\hline
\end{tabular}

TABLE 2: Difference analysis of abstinence cases and abstinence rate for all groups(N=78)

Notes: Patients withdrawn were considered treatment failures then the abstinence outcome includes 78 patients.

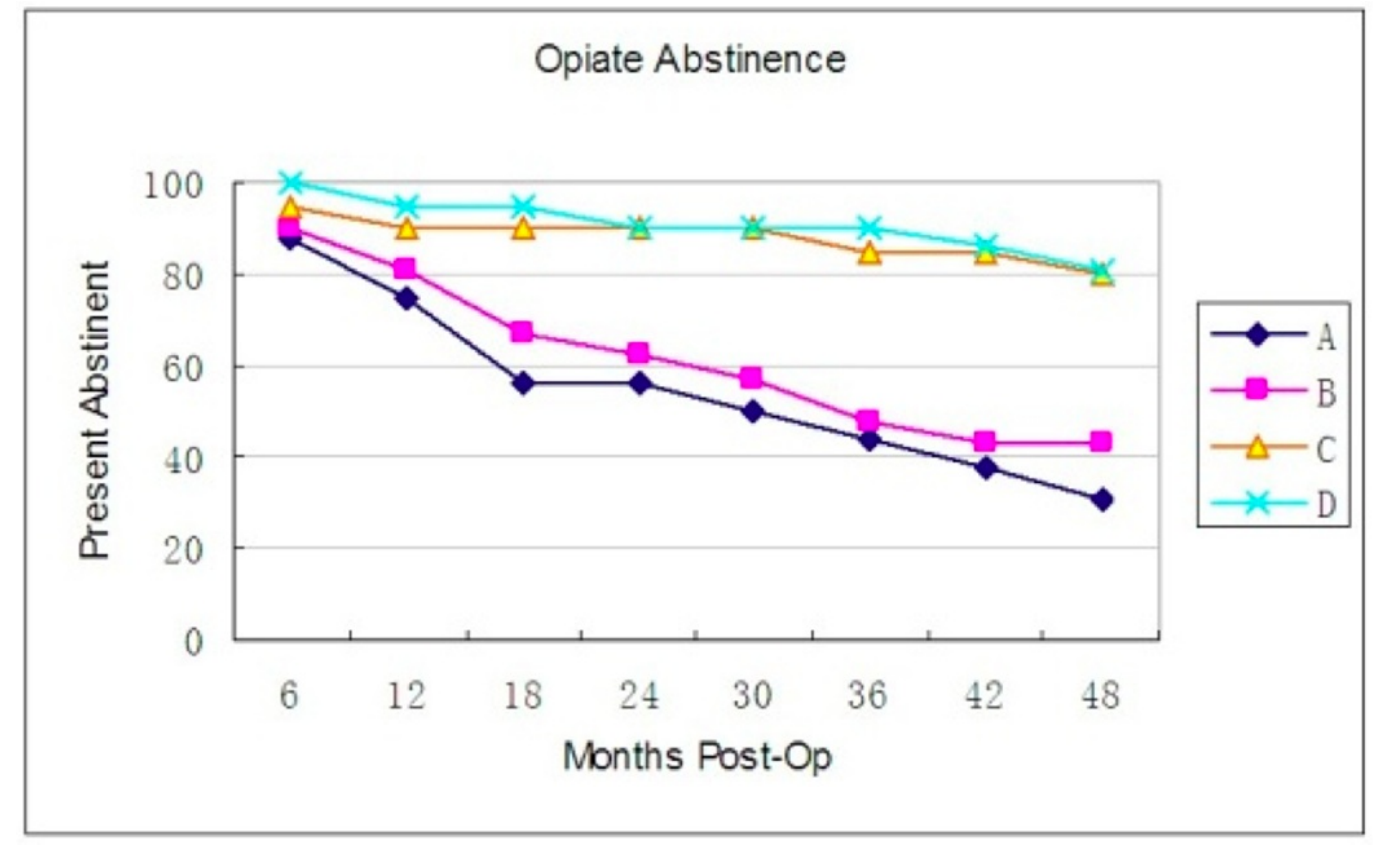

FIGURE 3: Rates of opioid abstinence over time.

Groups were followed at six month intervals. Follow-up included interviews with patient, interviews with family, urine drug screening, and naloxone pupil testing. Statistical analysis performed at 48 months post-op. The abstinence rates of Group C $(75.0 \%)$ and Group D $(76.2 \%)$ were significantly higher than Group A $(31.3 \%)$ and B $(28.6 \%)(p \leq 0.005)$.

Groups were followed at six month intervals. Follow-up included interviews with patient, interviews with family, urine drug screening, and naloxone pupil testing. Statistical analysis performed at 48 months postop. The abstinence rates of Group C (75.0\%) and Group D (76.2\%) were significantly higher than Group A $(31.3 \%)$ and B $(28.6 \%)(p \leqslant 0.005)$. 


\section{Cureus}

\section{Complications}

Several categories of complications and adverse events were tracked. Transient post-surgical adverse events included fever (14.5\%), nausea/emesis (5.3\%), seizure (1.3\%), and delirium (2.6\%). There were no infections or intracranial hemorrhages. However, there was a significant rate of neuropsychiatric adverse events. Typical complaints of patients with subjective complaints included "no goals for life and future", "no upward mobility", "being idle", and other complaints. In order to better assay these problems, during four years of follow-up, all patients underwent formal neuropsychiatric testing. Anterograde amnesia, motivational dysfunction, affective disorders, and dysosphresia were assessed using standardized exams. The total rate of neuropsychologic adverse events was $24.3 \%$ (18/74) (Tables 3, 4). While the number of patients may have been too small to detect differences, there was no significant difference for total specific complications rate between group A, B, C and D ( $\chi^{2}$-test 2.582, pख0.05).

The patients as a group in this research had statistical difference in CCCRC, 16PF-C, and MMPI-ANG between pre- and post-operation. Patients who were subjectively noted to have neuropsychiatric sequelae, whether identified by self, family, or clinician, demonstrated altered scores on all tests except EPQ-N (Table 5). If these patients are excluded from the analysis, there was no overall difference in neuropsychologic scores pre- and postoperatively.

Sexual function, to our knowledge, does not have proper scales for formal evaluation. We only ask the patients to report the changes of their sexual function pre- and postoperatively. Only three male patients $(4.1 \%, 3 / 74)$ reported changes pre- and postoperatively. Among them, two reported decline of sexual function, while one reported increase of sexual function. Considering the characteristics of China's traditional culture, the actual rate of sexual dysfunction may be higher.

\section{Social functions}

At the last interview, i.e. four years after the surgery, all the patients lived independently, $75.7 \%$ (56/74) of patients had been employed, while only $32.4 \%$ (24/74) of patients had been employed pre-operation. 64.9\% (48/74) of patients were completely financially independent, while only $17.6 \%(13 / 74)$ were independent pre-operatively. $73.0 \%(54 / 74)$ of the patients had become married or stayed married, while preoperatively, there were only $59.4 \%(44 / 74)$ married.

\begin{tabular}{|l|l|l|l|l|l|}
\hline Items & Group A & Group B & Group C & Group D & P \\
\hline Total patients & 16 & 19 & 19 & 20 & \\
Complications: patients and rate & $2(12.5 \% \square$ & $4(21.1 \% \square$ & $5(26.3 \% \square$ & $7(35.0 \% \square$ & $>0.05$ \\
\hline
\end{tabular}

TABLE 3: Difference analysis of complications cases and complications rate for all groups $(\mathrm{N}=\mathbf{7 4})$ 


\section{Cureus}

\begin{tabular}{|c|c|c|c|c|c|}
\hline Complications & Group A & Group B & Group C & Group D & Total \\
\hline Anterograde amnesia & 1 & 0 & 1 & 1 & $3(4.1 \%)$ \\
\hline Motivation dysfunction & 2 & 0 & 3 & 4 & $9(12.2 \%)$ \\
\hline Affective disorder & 0 & 3 & 2 & 2 & $7(9.5 \%)$ \\
\hline Dysosphresia & 0 & 2 & 1 & 1 & $4(5.4 \%)$ \\
\hline
\end{tabular}

\section{TABLE 4: Long-term neuropsychiatric adverse events by group $(n=74)$}

Note: Each adverse event was counted as an isolated incident. Thus, it was possible for one patient to have multiple adverse events. Events were assessed both by subjective impression and clinical rating scale.

\begin{tabular}{|c|c|c|c|c|c|c|c|c|}
\hline Event(test) & $\mathbf{n}$ & Pre & Post & $\mathbf{P}$ & $\mathbf{n}$ & Pre & Post & $\mathbf{P}$ \\
\hline CCCRC & 74 & $6.19 \pm 0.69$ & $5.98 \pm 0.65$ & 0.041 & 4 & $6.56 \pm 0.24$ & $4.50 \pm 0.20$ & 0.002 \\
\hline WMS-RC & 74 & $82.80 \pm 7.35$ & $81.91 \pm 7.38$ & 0.267 & 3 & $81.00 \pm 8.19$ & $62.00 \pm 5.57$ & 0.042 \\
\hline Ac & 74 & $7.28 \pm 2.08$ & $6.78 \pm 2.21$ & 0.063 & 9 & $7.22 \pm 0.67$ & $3.67 \pm 1.00$ & $\square 0.001$ \\
\hline Cs & 74 & $15.43 \pm 2.35$ & $14.96 \pm 2.02$ & 0.086 & 9 & $15.33 \pm 0.87$ & $12.00 \pm 0.71$ & $\square 0.001$ \\
\hline EPQ-N & 74 & $64.91 \pm 6.81$ & $63.73 \pm 7.43$ & 0.229 & 7 & $59.14 \pm 13.70$ & $70.14 \pm 5.01$ & 0.057 \\
\hline 16PF-C & 74 & $4.09 \pm 1.43$ & $3.64 \pm 1.43$ & 0.015 & 7 & $4.14 \pm 0.69$ & $3.14 \pm 0.69$ & 0.004 \\
\hline MMPI-ANG & 74 & $52.82 \pm 6.66$ & $55.22 \pm 7.81$ & $<0.001$ & 7 & $55.57 \pm 2.82$ & $70.43 \pm 6.16$ & $\square 0.001$ \\
\hline
\end{tabular}

TABLE 5: Pre- and postop neuropsychiatric scores (Mean \pm Std.) Table 5-A total patients | Table 5-B patients with subjective adverse events

\section{Discussion}

This study reports the first randomized, blind, prospective clinical trial for the treatment of psychological heroin dependence by means of stereotactic ablation of the NAc. It identifies specific lesion locations within the NAc that appear to optimize its efficacy. The data above presents credible evidence for the success of lesioning of the posteromedial NAc in eliminating psychological opiate addiction. The principal finding is that a specific area of lesioning (i.e., including both the anterior and lateral NAc as in Groups C and D) produced a significantly more reliable extinction of addiction. This benefit appears long-term, with nearly $75 \%$ of patients free from opioid addiction four years later while receiving no other treatments. No other known therapy has reported this degree of efficacy and durability.

Although the benefits of NAc lesioning are notable, any therapeutic intervention requires careful risk-benefit analysis. While there were no infections or hemorrhages with treatment, 
psychological morbidity was not uncommon. Dysophresia, anterograde amnesia, affective disorders, and motivation dysfunction occurred in a significant number of patients. Some of these effects may stem from injury to structures surrounding the NAc (e.g., olfactory cortex damage can cause dysosphresia; damage to the orbitofrontal cortex could underlie motivation dysfunction, as well as memory and affective disorders). However, some of these neuropsychological phenomena may be directly related to the intentional damage to NAc. For example, stimulation of ventral portions of the NAc may alter mood [12]. This could prove to be an intrinsic limitation to the proposed procedure; while technical improvements might decrease collateral damage and associated psychological complications, some deleterious sideeffects may be unavoidable with this specific technique.

Several critical questions remain unanswered by the current study. Principally, the neuropsychological risks to patients, and ways to limit them, require more intense investigation. Moreover, it remains unclear whether the posteromedial NAc needs to be ablated in humans for efficacy, or whether only the lateral and anterior portions are sufficient. In addition, the conditions of the experimental therapy denied patients access to counseling postoperatively, a condition that is unlikely to exist in actual practice. How this would alter results is unclear; however, the benefit experienced by patients without counseling is notable. Finally, the issue of informed consent in the setting of criminalized addiction is ethically treacherous. Informed consent in such a setting implies that patients' desire to abstain from heroin, which, while within the specific setting of a regulated study may be carefully noted, the same cannot be assumed of those who are compelled to undergo treatment. However, these issues are not specific to surgical interventions, as they apply to all interventions to treat addictions; though in surgical settings, the question is most acute and requires special attention.

This research spurs several questions that might be answered by further animal and human research. For example, additional work can be performed on the phenomenology of addiction in these patients, and in particular, which portion of addictive behavior is absent in these patients. Furthermore, numerous questions remain regarding the effects of lesioning on the diverse functions of the nucleus accumbens on the molecular, cellular, synaptic, metabolic, phenomenologic, and functional levels. Further work may suggest ways to limit lesion size, and thereby reduce its neuropsychologic morbidity. In addition, other methods currently in practice, including deep brain stimulation and stereotactic radiosurgery, may offer safer techniques not only of lesioning, but of modulating NAc activity, and might prove to be less risky interventions. Newer techniques of neuromodulation of the NAc, such as optogenetics, which may allow for cell-selective interventions, demonstrate some efficacy in animal models [42] and may help limit adverse effects.

The history of surgery for psychiatric indications is fraught with medical zealotry and abrogation of patients' rights. However, in the face of so much contemporary suffering that stems from the human predilection for substance abuse, it is equally troubling that modern medicine may be trapped by history's missteps. The lack of reliable treatment to debilitating disease does not give an ethical carte-blanche to any intervention, but it does clarify the urgency of an ethical imperative to identify safe and effective treatment. Studying present and past errors, such as frontal leucotomy, may help deter future wrongs. Foremost among the lessons learned are the need for establishing, at the outset, effective metrics for both outcomes and adverse events. Randomized prospective data, such as those presented here, will be vital in understanding surgical treatment of psychiatric disease going forward.

\section{Conclusions}

It is critical that special consideration be paid to the indications of these surgical techniques for addressing behavioral disorders. Strict attention to the patient's compelling interest, rather 
than society's, in controlling disruptive or illegal behavior must be foremost. With this understanding, we acknowledge the importance of adherence, at a minimum, to the Belmont principles. Detailed informed consent is necessary, even while acknowledging the complicated nature of agency in addiction and its treatment. Interventions into the nucleus accumbens may alter a patient's agency, but this must be counterbalanced with the knowledge that psychiatric illness can rob patients of this same agency. It is hoped that multidisciplinary public dialogues, both secular and academic, may help foster the ethical use of these promising invasive behavioral interventions.

Drug addiction challenges patients, families, physicians, and society at large. Despite the magnitude of the problem, few reliable therapies exist. Substance abuse is generally deemed to be incurable, requiring long-term maintenance therapy, with frequent relapses. Perhaps the data presented here, with significant future study, may one day yield an early a cure for this most human of afflictions.

\section{Additional Information}

\section{Disclosures}

Human subjects: Consent was obtained by all participants in this study. The Ethics Committee of Fourth Military Medical University issued approval N/A. Animal subjects: All authors have confirmed that this study did not involve animal subjects or tissue. Conflicts of interest: In compliance with the ICMJE uniform disclosure form, all authors declare the following:

Payment/services info: Funding sources: (1) China National "11th five years" scientific support plan project $\mathbf{X}$ Clinical reevaluation of current prevention of relapse区 ХNo.2007BAI0703区 (2) Scientific and technological achievements popularizing plan project supported by China, Shaanxi Province Government in the year 2005 (No.2005KT-027) (3) Major clinical high-technique program $\mathbf{X C l i n i c a l}$ and experimental research on stereotactic neurosurgery for treating addiction Xgranted by the health subdepartment of General Logistics Department of Peoples Liberation Army (No.200218) The funders had no role in study design, data collection and analysis, decision to publish, or preparation of the manuscript. Trials registered number ISRCTN33954423. Financial relationships: All authors have declared that they have no financial relationships at present or within the previous three years with any organizations that might have an interest in the submitted work. Other relationships: All authors have declared that there are no other relationships or activities that could appear to have influenced the submitted work.

\section{Acknowledgements}

Acknowledgements: We express our gratitude to every patient for participating in this study. Furthermore, we thank Xingsuo Wang and Tian Luo for their close follow-up with all patients. Moreover, Drs. Qing Li and Wei Wang deserve our thanks for obtaining and processing all patient MRIs. Lastly, we wish to express our sincere appreciation to Dr. Paul Kalanithi for his immensely helpful conversations voicing his sincere ethical concerns, his encouragement to present complications, and advice on appropriate data analysis and interpretation. Contributions: Xuelian Wang and Guodong Gao are responsible for the design of this study. Chongwang Chang, Shunnan Ge, Nan Li, Ning Geng, Jing Wang, and Xin Wang devoted themselves to data collection and paper writing. John R Adler is in charge of data handling and polishing the language.

\section{References}

1. Amato L, Minozzi S, Davoli M, Vecchi S, Ferri MM, et al.: Psychosocial and pharmacological treatments versus pharmacological treatments for opioid detoxification. Cochrane Database Syst Rev. 2008, 4:CD005031 
2. Faggiano F, Vigna-Taglianti F, Versino E, Lemma P: Methadone maintenance at different dosages for opioid dependence. Cochrane Database Syst Rev. 2003, 3: CD002208

3. Sees KL, Delucchi KL, Masson C, Rosen A, Clark HW, et al.: Methadone maintenance vs 180day psychosocially enriched detoxification for treatment of opioid dependence: a randomized controlled trial. JAMA. 2000, 283:1303-1310. 10.1001/jama.283.10.1303

4. Tang YL, Zhao D, Zhao C, Cubells JF: Opiate addiction in China: current situation and treatments. Addiction. 2006, 101:657-665. 10.1111/j.1360-0443.2006.01367.x

5. Schottenfeld RS, Chawarski MC, Mazlan M: Maintenance treatment with buprenorphine and naltrexone for heroin dependence in Malaysia: a randomised, double-blind, placebocontrolled trial. Lancet. 2008, 371:2192-2200. 10.1016/S0140-6736(08)60954-X

6. Salamina G, Diecidue R, Vigna-Taglianti F, Jarre P, Schifano P, et al.: Effectiveness of Therapies for Heroin Addiction in Retaining Patients in Treatment: Results From the VEdeTTE Study. Subst Use Misuse. 2010, 45:2076-2092. 10.3109/10826081003791932

7. Balfour DJ: Neuroplasticity within the mesoaccumbens dopamine system and its role in tobacco dependence. Curr Drug Targets CNS Neurol Disord. 2002, 1:413-421.

8. Groenewegen HJ, Wright CI, Beijer AV: The nucleus accumbens: gateway for limbic structures to reach the motor system?. Prog Brain Res. 1996, 107:485-511.

9. Boileau I, Assaad JM, Pihl RO, Benkelfat C, Leyton M, et al.: Alcohol promotes dopamine release in the human nucleus accumbens. Synapse. 2003, 49:226-231. 10.1002/syn.10226

10. Carelli RM: Nucleus accumbens cell firing during goal-directed behaviors for cocaine vs. 'natural' reinforcement. Physiol Behav. 2002, 76:379-387.

11. Gao G, Wang X, He S, Li W, Wang Q, et al.: Clinical study for alleviating opiate drug psychological dependence by a method of ablating the nucleus accumbens with stereotactic surgery. Stereotact Funct Neurosurg. 2003, 81:96-104. 75111

12. Okun MS, Mann G, Foote KD, Shapira NA, Bowers D, et al.: Deep brain stimulation in the internal capsule and nucleus accumbens region: responses observed during active and sham programming. J Neurol Neurosurg Psychiatry. 2007, 78:310-314. 10.1136/jnnp.2006.095315

13. Bewernick BH, Hurlemann R, Matusch A, Kayser S, Grubert C, et al.: Nucleus accumbens deep brain stimulation decreases ratings of depression and anxiety in treatment-resistant depression. Biol Psychiatry. 2010, 67:110-116. 10.1016/j.biopsych.2009.09.013

14. Huff W, Lenartz D, Schormann M, Lee SH, Kuhn J, et al.: Unilateral deep brain stimulation of the nucleus accumbens in patients with treatment-resistant obsessive-compulsive disorder: Outcomes after one year. Clin Neurol Neurosurg. 2010, 112:137-143.

10.1016/j.clineuro.2009.11.006

15. Kuhn J, Bauer R, Pohl S, Lenartz D, Huff W: Observations on unaided smoking cessation after deep brain stimulation of the nucleus accumbens. Eur Addict Res. 2009, 15:196-201. $10.1159 / 000228930$

16. Kuhn J, Lenartz D, Huff W, Lee S, Koulousakis A, et al.: Remission of alcohol dependency following deep brain stimulation of the nucleus accumbens: valuable therapeutic implications?. J Neurol Neurosurg Psychiatry. 2007, 78:1152-1153. 10.1136/jnnp.2006.113092

17. Muller UJ, Sturm V, Voges J, Heinze HJ, Galazky I, et al.: Successful treatment of chronic resistant alcoholism by deep brain stimulation of nucleus accumbens: first experience with three cases. Pharmacopsychiatry. 2009, 42:288-291. 10.1055/s-0029-1233489

18. Neuner I, Podoll K, Lenartz D, Sturm V, Schneider F: Deep brain stimulation in the nucleus accumbens for intractable Tourette's syndrome: follow-up report of 36 months. Biol Psychiatry. 2009, 65:e5-e6. 10.1016/j.biopsych.2008.09.030

19. Sturm V, Lenartz D, Koulousakis A, Treuer H, Herholz K, et al.: The nucleus accumbens: a target for deep brain stimulation in obsessive-compulsive- and anxiety-disorders. J Chem Neuroanat. 2003, 26:293-299.

20. Tass PA, Klosterkotter J, Schneider F, Lenartz D, Koulousakis A, et al.: Obsessive-compulsive disorder: development of demand-controlled deep brain stimulation with methods from stochastic phase resetting. Neuropsychopharmacology. 2003, 28 Suppl 1:S27-S34.

10.1038/sj.npp.1300144

21. van Kuyck K, Gabriels L, Cosyns P, Arckens L, Sturm V, et al.: Behavioural and physiological effects of electrical stimulation in the nucleus accumbens: a review. Acta Neurochir Suppl. 2007, 97(Pt 2):375-391.

22. Wang B, Luo F, Ge XC, Fu AH, Han JS: Effects of lesions of various brain areas on drug priming or footshock-induced reactivation of extinguished conditioned place preference. Brain Res. 
2002, 950:1-9.

23. He F, Guan H, Zhao Z, Miao X, Zhou Q, et al.: Evaluation of short-term psychological functions in opiate addicts after ablating the nucleus accumbens via stereotactic surgery. Stereotact Funct Neurosurg. 2008, 86:320-329. 10.1159/000160155

24. Qiu J: China clamps down on controversial therapies . Lancet. 2009, 373:1834-1835.

25. Jacobs EH, Smit AB, de Vries TJ, Schoffelmeer AN: Long-term gene expression in the nucleus accumbens following heroin administration is subregion-specific and depends on the nature of drug administration. Addict Biol. 2005, 10:91-100. 10.1080/13556210412331284748

26. D'Este L, Scontrini A, Casini A, Pontieri FE, Renda TG: Heroin sensitization as mapped by cFos immunoreactivity in the rat striatum. Brain Res. 2002, 933:144-149.

27. Jacobs EH, Spijker S, Verhoog CW, Kamprath K, de Vries TJ, et al: Active heroin administration induces specific genomic responses in the nucleus accumbens shell. FASEB J. 2002, 16:19611963. 10.1096/fj.02-0272fje

28. Nikoshkov A, Drakenberg K, Wang X, Horvath MC, Keller E, et al.: Opioid neuropeptide genotypes in relation to heroin abuse: dopamine tone contributes to reversed mesolimbic proenkephalin expression. Proc Natl Acad Sci USA. 2008, 105:786-791.

10.1073/pnas.0710902105

29. Owesson-White CA, Ariansen J, Stuber GD, Cleaveland NA, Cheer JF, et al.: Neural encoding of cocaine-seeking behavior is coincident with phasic dopamine release in the accumbens core and shell. Eur J Neurosci. 2009, 30:1117-1127. 10.1111/j.1460-9568.2009.06916.x

30. Hollander JA, Carelli RM: Cocaine-associated stimuli increase cocaine seeking and activate accumbens core neurons after abstinence. J Neurosci. 2007, 27:3535-3539. 10.1523/ JNEUROSCI.3667-06.2007

31. Martin M, Chen BT, Hopf FW, Bowers MS, Bonci A: Cocaine self-administration selectively abolishes LTD in the core of the nucleus accumbens. Nat Neurosci. 2006, 9:868-869. 10.1038/nn1713

32. Calu DJ, Schoenbaum G: Cocaine-paired cues activate aversive representations in accumbens neurons. Neuron. 2008, 57:633. 10.1016/j.neuron.2008.02.025

33. Suto N, Ecke LE, Wise RA: Control of within-binge cocaine-seeking by dopamine and glutamate in the core of nucleus accumbens. Psychopharmacology (Berl). 2009, 205:431-439. 10.1007/s00213-009-1553-0

34. Mitrovic I, Napier TC: Mu and kappa opioid agonists modulate ventral tegmental area input to the ventral pallidum. Eur J Neurosci. 2002, 15:257-268.

35. Ward HG, Nicklous DM, Aloyo VJ, Simansky KJ: Mu-opioid receptor cellular function in the nucleus accumbens is essential for hedonically driven eating. Eur J Neurosci. 2006, 23:16051613. 10.1111/j.1460-9568.2006.04674.x

36. Simmons D, Self DW: Role of mu- and delta-opioid receptors in the nucleus accumbens in cocaine-seeking behavior. Neuropsychopharmacology. 2009, 34:1946-1957. 10.1038/npp.2009.28

37. Xiong $\mathrm{W}$, Yu LC: Involvements of mu- and kappa-opioid receptors in morphine-induced antinociception in the nucleus accumbens of rats. Neurosci Lett. 2006, 399:167-170. 10.1016/j.neulet.2006.01.052

38. Hirose N, Murakawa K, Takada K, Oi Y, Suzuki T, et al: Interactions among mu- and deltaopioid receptors, especially putative delta1- and delta2-opioid receptors, promote dopamine release in the nucleus accumbens. Neuroscience. 2005, 135:213-225.

10.1016/j.neuroscience.2005.03.065

39. Soderman AR, Unterwald EM: Cocaine reward and hyperactivity in the rat: sites of mu opioid receptor modulation. Neuroscience. 2008, 154:1506-1516. 10.1016/j.neuroscience.2008.04.063

40. Walker JR, Ahmed SH, Gracy KN, Koob GF: Microinjections of an opiate receptor antagonist into the bed nucleus of the stria terminalis suppress heroin self-administration in dependent rats. Brain Res. 2000, 854:85-92.

41. Wang J, Zhao Z, Liang Q, Wang X, Chang C, et al: The nucleus accumbens core has a more important role in resisting reactivation of extinguished conditioned place preference in morphine-addicted rats. J Int Med Res. 2008, 36:673-681.

42. Witten IB, Lin SC, Brodsky M, Prakash R, Diester I, Anikeeva P, Gradinaru V, Ramakrishnan C, and Deisseroth K: Cholinergic Interneurons Control Local Circuit Activity and Cocaine Conditioning. Science. 2010, 330:1677-1681. 10.1126/science.1193771 